\title{
Die Grenzen des Sozialraums
}

\section{Gemeinwesenorientierte Jugendarbeit am Beispiel "Platzmanagement Alexanderplatz" in Berlin}

\section{CLAUDIA STECKELBERG}

Prof. Dr. Claudia Steckelberg lehrt an der Dualen Hochschule Stuttgart die Arbeitsschwerpunkte Theorien und Methoden Sozialer Arbeit,

Gemeinwesenarbeit, Wohnungslosenhilfe, Jugend(sozial)arbeit und Geschlechterforschung.

E-Mail steckelberg@dhbw-stuttgart.de

\author{
Mobile Jugendarbeit wird oft nur in Stadtteilen \\ angeboten, die als "problematisch" gelten. Vor \\ Herausforderungen steht die Jugendarbeit, wenn \\ sie sich auf städtische Räume bezieht, die von \\ besonderer touristischer und wirtschaftlicher \\ Bedeutung sind, wie ein Beispiel aus Berlin zeigt.
}

Touristischer Anziehungspunkt, Verkehrsknotenpunkt des Nahverkehrs, Shopping Malls und Einzelhandelsketten, Treffpunkt für Menschen unterschiedlichen Alters und Herkunft, Wohnort - all dies (und noch viel mehr) macht die Attraktivität des Alexanderplatzes im Berliner Bezirk Mitte aus.

Als städtischer Platz von historischer und aktueller nationaler sowie auch internationaler Bekanntheit treffen hier eine Vielfalt von Menschen und unterschiedliche Nutzungsinteressen aufeinander. Als Alexanderplatz wird nicht nur der im Stadtplan als solcher ausgewiesene Platz mit der Weltzeituhr bezeichnet - auch die Anlagen, Häuser und Geschäfte rund um den Fernsehturm werden von den Nutzerinnen und Nutzern »der Alex« genannt.

\section{Ein »bunter Ort» für junge Menschen}

Unter diesen Nutzergruppen findet sich eine erhebliche Anzahl junger Menschen. In einer Erhebung zur Nutzung des Sozialraums aus Sicht junger Menschen (Homann/Steckelberg 2010) wurde deutlich, dass für Jugendliche, die Berlin bereisen, der Alexanderplatz einer der ersten Besuchsorte ist.

Als Treffpunkt nutzen den zentralen städtischen Platz jeden Tag im Schnitt circa 500 junge Menschen aus vielen Berliner Bezirken und Brandenburg mit unterschiedlichen Motiven: zum "Abhängen « nach der Schule, als Treff- punkt, für sportliche Aktivitäten, zum Einkaufen oder als Ausgangsort für das nächtliche Feiern am Wochenende.

Nur ein verschwindend geringer Teil dieser jungen Menschen wohnt auch in der Nähe des Alexanderplatzes. Nach den Vorzügen gefragt, bezeichnen viele junge Menschen den Alexanderplatz als »bunten Ort «, an dem sie Gleichgesinnte treffen und auch neue interessante Menschen kennenlernen können. Gerade Jugendliche mit einem auffälligeren äußeren Erscheinungsbild (Gothics, Punks, aber auch solche, die sich nicht einer Szene zuordnen lassen wollen) beklagen vielfach Diskriminierungen in ihrem Wohnumfeld und erfahren mitten in der Großstadt eher Akzeptanz. Vor allem rund um den Fernsehturm wird der Platz nicht nur als Treffpunkt, sondern als Ort der Präsentation im öffentlichen Raum genutzt. Neben dieser bunten Vielfalt begrüßen die jungen Menschen den Alexanderplatz als einen Ort jenseits pädagogischer Aufsicht und Vorgaben, die sie in der Regel in Jugendzentren vorfinden. (Homann/ Steckelberg 2010: 4-7)

Trotz der offensichtlichen Präsenz junger Menschen ist der Alexanderplatz ein eher untypischer Ort für Jugendarbeit im öffentlichen Raum. Zwar gehört es zum Selbstverständnis lebensweltorientierter Jugendarbeit, junge Menschen an ihren Aufenthaltsorten aufzusuchen und von dort aus gemeinsam mit Einzelnen oder auch Cliquen Angebote und Projekte zu entwickeln. Doch zumeist 
wird diese mobile Jugendarbeit in Stadtteilen angeboten, die aus unterschiedlichen Gründen als problematisch gelten und vor allem dort, wo junge Menschen durch ihren Aufenthalt auf der Straße und durch ihre Nutzung des öffentlichen Raums auffallen.

Vor Herausforderungen steht die Jugendarbeit, wenn sie sich auf städtische Räume bezieht, die von besonderer touristischer und wirtschaftlicher Bedeutung sind. Hier ist Soziale Arbeit häufig nur gefragt, um sich um unerwünschte Nutzerinnen und Nutzer, darunter auch junge Menschen, zu kümmern, die das erwünschte Stadtbild stören. attraktiver Begegnungsort für junge Menschen und Familien zu erhalten sei.

Die Tragweite dieser Entscheidung wird deutlich, wenn man sich vor Augen führt, dass der Alexanderplatz seit Jahrzehnten und auf nicht absehbare Zeit Ort weitreichender baulicher Veränderungen ist und sein wird. Bei solchen städtebaulichen Entwicklungen geraten soziale Aspekte gegenüber architektonischen und wirtschaftlichen Erwägungen leicht ins Hintertreffen.

Gabriele Bingel (2008) weist darauf hin, dass sich Jugendliche im öffentlichen Raum in einem Spannungsfeld zwischen Raumnahme und Raumzu-

"Die Annahme, Menschen hätten

in ihrem Wohnumfeld auch ihren

Lebensmittelpunkt, trifft nicht immer zu«

\section{Platzmanagement Alexanderplatz als gemeinwesenorientierte Jugendarbeit}

Junge Menschen nicht als (potenziellen) Störfaktor im urbanen Raum anzusehen, sondern als erwünschte Nutzerinnen und Nutzer - aus dieser Leitidee entstand das Projekt »Platzmanagement Alexanderplatz « in Berlin. Entscheidende Grundlage war dabei ein Beschluss der Bezirksverordnetenversammlung von Berlin-Mitte im Jahr 1998, der besagt, dass der Alexanderplatz als weisung bewegen (ebd., 98). »Raumnahme wird nur toleriert, solange sie den jeweiligen normativen Deutungen der Erwachsenengeneration entspricht. Das Spannungsverhältnis zwischen Raumaneignung Jugendlicher und gesellschaftlicher Raumzuweisung zeigt, wie Gestaltungsspielräume junger Menschen mit den gesellschaftlichen Rahmenbedingungen und Jugendbildern widersprüchlich verwoben sind." (ebd., 99) Aneignungsprozesse junger Menschen verlaufen deshalb vielfach konflikthaft, weil sie sich im Widerstand gegen eingeschränkte Handlungsspielräume und herrschende Deutungsmacht bewegen (vgl. ebd., 100).

Um unter diesen Bedingungen den Beschluss der Bezirksverordnetenversammlung umsetzen zu können, wurde im Jahre 2000 das Platzmanagement Alexanderplatz gegründet. Anders als die meisten Einrichtungen der Jugendarbeit bietet das Projekt seit Beginn keine eigenen Räume als Treffpunkt für junge Menschen, sondern konzentriert sich auf den öffentlichen Raum. Seit 2010 arbeitet das Platzmanagement Alexanderplatz unter der Trägerschaft des Moabiter Ratschlag e. V., einem Verein, bei dem Gemeinwesenorientierung und Kinder- und Jugendbeteiligung wesentliche Arbeitsschwerpunkte darstellen.

Der Name »Platzmanagement Alexanderplatz « verursacht immer wieder Missverständnisse. Vielfach wird vermutet, es handele sich dabei um eine Einrichtung für die Belange aller Bewohnerinnen und Bewohner und für alle Akteure im Sozialraum, vergleichbar mit einem Stadtteilbüro. Tatsächlich ist das Platzmanagement jedoch in der Jugendarbeit nach $\mathbb{S} 11$ SGB VIII verortet mit einer dadurch klar umrissenen Zielgruppe.

Dennoch ist die Nähe zur Stadtteilund Gemeinwesenarbeit, die mit dem Begriff Platzmanagement transportiert wird, ein unverzichtbarer Aspekt von Jugendarbeit im öffentlichen Raum. Denn diese kann nur gelingen, wenn hierfür in Zusammenarbeit mit anderen Akteuren Akzeptanz und geeignete Bedingungen geschaffen werden. Inhaltlich geht es darum, die Aufenthaltsqualität für junge Menschen auf dem Alexanderplatz zu erhalten und

\section{$\S 11$ Jugendarbeit}

(1) Jungen Menschen sind die zur Förderung ihrer Entwicklung erforderlichen Angebote der Jugendarbeit zur Verfügung zu stellen. Sie sollen an den Interessen junger Menschen anknüpfen und von ihnen mitbestimmt und mitgestaltet werden, sie zur Selbstbestimmung befähigen und zu gesellschaftlicher Mitverantwortung und zu sozialem Engagement anregen und hinführen.

(2) Jugendarbeit wird angeboten von Verbänden, Gruppen und Initiativen der Jugend, von anderen Trägern der Jugendarbeit und den Trägern der öffentlichen Jugendhilfe. Sie umfasst für Mitglieder bestimmte Angebote, die offene Jugendarbeit und gemeinwesenorientierte Angebote.
(3) Zu den Schwerpunkten der Jugendarbeit gehören:

1. außerschulische Jugendbildung mit allgemeiner, politischer, sozialer, gesundheitlicher, kultureller, naturkundlicher und technischer Bildung,

2. Jugendarbeit in Sport, Spiel und Geselligkeit,

3. arbeitswelt-, schul- und familienbezogene Jugendarbeit,

4. internationale Jugendarbeit,

5. Kinder- und Jugenderholung,

6. Jugendberatung.

(4) Angebote der Jugendarbeit können auch Personen, die das 27. Lebensjahr vollendet haben, in angemessenem Umfang einbeziehen. 


\section{Stichwort: Mobile Jugendarbeit}

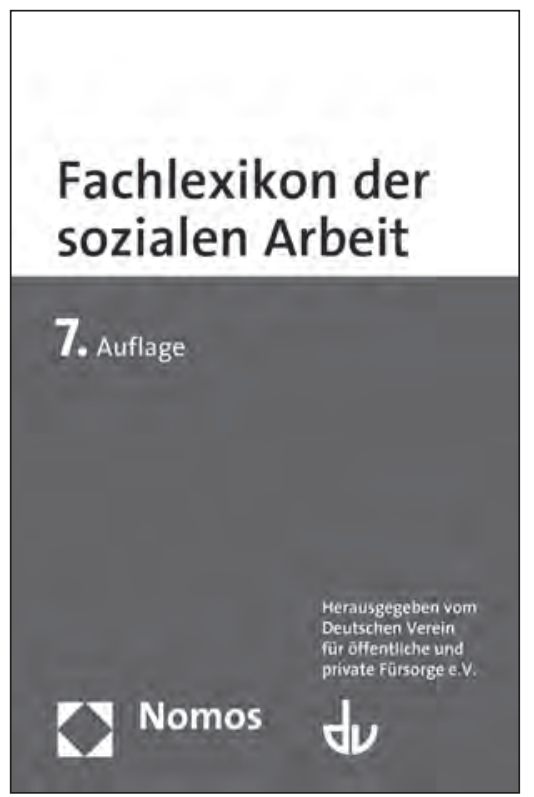

Quelle: Deutscher Verein für öffentliche und private Fürsorge e. V. (Hg.): Fachlexikon der sozialen Arbeit. 7. Auflage. Nomos Verlagsgesellschaft, BadenBaden 2011. Ca. 1.200 Seiten. 44,- Euro. ISBN 978-3-8329-5153-5.
Im Feld der Jugendhilfe hat sich die aus dem angloamerikanischen Raum stammende Arbeitsform Streetwork durch konzeptionelle Erweiterung und praxisbezogene Ausdifferenzierung zur Mobilen Jugendarbeit entwickelt. Zu Beginn der 1970er-Jahre zunächst regional auf den baden-württembergischen Raum konzentriert, hat sich Mobile Jugendarbeit inzwischen bundesweit etabliert. Die konzeptionellen und arbeitspraktischen Grenzen zu Streetwork, zu hinausreichender Jugendarbeit (= outreach work), zu sozialraumorientierter Jugendarbeit und zu Gassenarbeit (insbesondere in der Schweiz) sind fließend: Spezifische Schwerpunkte liegen bei Mobiler Jugendarbeit im Allgemeinen jedoch auf einer weniger szene-/brennpunktbezogenen Ausrichtung und einem stärker stadtteilorientierten/sozialräumlichen Fokus sowie auf der Verbindung zwischen lebensweltzentriertem Aufsuchen und einrichtungsgebundener Club-/Freizeitarbeit. Die überspitzten konzeptionellen Abgrenzungsdiskurse der 1970er- und 1980er-Jahre haben sich in den 1990erJahren zu einem produktiven Diskussions- zusammenhang entwickelt, der schließlich zur Gründung einer übergreifenden fachverbandlichen Struktur in Form der Bundesarbeitsgemeinschaft Streetwork/ Mobile Jugendarbeit führte (http://www. bundesarbeitsgemeinschaftstreetworkmobile-jugendarbeit.de). Forciert durch die in Stuttgart ansässige »International Society for Mobile Youth Work (ISMO)« finden die Leitideen Mobiler Jugendarbeit mittlerweile verstärkt Resonanz auf internationaler Ebene wie etwa im Zusammenhang mit dem Aufbau von Jugendhilfestrukturen in Osteuropa, aber auch in der Entwicklung gemeinwesennaher Projekte zur Arbeit mit Straßenkindern in Asien, Afrika und Süd- und Mittelamerika.

Werner Steffan

Prof. Dr. Werner Steffan ist Hochschullehrer für Sozialarbeit und Sozialpädagogik, insbesondere Beratung, Unterstützung, Information und Öffentlichkeitsarbeit an der Fachhochschule Potsdam. durch gestalterische und bauliche Maßnahmen zu fördern. Dies gilt auch für Aktivitäten in den Bereichen Musik, Bewegung, Sport, die zwar durch die Jugendarbeit unterstützt werden, jedoch andererseits von anderen Nutzerinnen und Nutzern unter Umständen als störend empfunden werden.

Hier schließt als weiterer Arbeitsschwerpunkt des Platzmanagements die Interessensvertretung junger Menschen in unterschiedlichen politischen Gremien und sozialen Zusammenhängen an. Dabei wird Vorurteilen gegenüber jungen Menschen entgegengewirkt und die Verständigung verschiedener Akteure befördert.

Im aktuell dominanten Diskurs zum Thema Sicherheit im öffentlichen Raum muss sich gemeinwesenorientierte Jugendarbeit einmischen. Es gilt ein Verständnis von Sicherheit durchzusetzen, das junge Menschen nicht als potenzielle Bedrohung von Sauberkeit und Ordnung ansieht, sondern das ihnen einen verlässlichen Schutz vor der Vertreibung von öffentlichen Plätzen gewährleistet.

Projekte im Verständnis non-formaler Bildungsarbeit, als weiterer Arbeits- schwerpunkt des Platzmanagements in enger Kooperation mit anderen Einrichtungen und Institutionen, werden bewusst so konzipiert, dass sie im Besonderen, aber nicht ausschließlich junge Menschen ansprechen. Im Jahr 2011 geschah dies beispielsweise in Form einer Aktion mit dem Titel »Alex wählt", bei der anlässlich der Bezirks- und Abgeordnetenhauswahlen in Berlin mit kreativen und interaktiven Methoden Demokratie und politische Interessensvertretung thematisiert wurden.

\section{Die Grenzen der sozialraumorientierten Logik in der Jugendarbeit}

In der Systematik der Sozialraumorientierung als Organisationsprinzip der Jugendhilfe in Berlin gehört der Alexanderplatz zum »Planungsraum Alexanderplatzviertel «, der wiederum ein Teil der weiträumigeren Bezirksregion Alexanderplatz ist. Wie auch in anderen Städten üblich, sind diese Planungsraum genannten Sozialräume geografisch klar begrenzte Einheiten, die als Grundlage für die Festlegung des Bedarfs an Ange- boten und Maßnahmen der Kinder- und Jugendhilfe dienen.

Für diesen Zweck werden ausgehend von den im Sozialraum wohnenden Menschen umfangreiche sozialstatistische Daten erhoben. Eine solche kleinräumige Planung soll es ermöglichen, der spezifischen Situation in den Sozialräumen gezielt und adäquat begegnen zu können. Als problematisch ist es anzusehen, dass in dieser Logik unter einem Sozialraum eine in Quadratmetern messbare räumliche Einheit verstanden wird (Reutlinger 2005: 88), der bestimmte Eigenschaften zugeschrieben werden.

Christian Reutlinger bezeichnet diese Logik als »Verdinglichung des Sozialraums « (ebd.), in der die lebensweltlichen Perspektiven der Betreffenden keinen Platz haben. Die Annahme, dass Menschen in ihrem räumlichen Wohnumfeld auch ihren Lebensmittelpunkt innehaben, entspricht nur zum Teil deren Lebenswelt. Ursula Nissen hat bereits 1998 auf die zunehmende Verinselung der Lebenswelten von Kindern und Jugendlichen verwiesen, die eben nicht auf ein geografisches Gebiet 
einzugrenzen seien, sondern je nach Lebensbereichen unterschiedliche Räume einbeziehen (Nissen 1998: 167).

Dass sich Jugendliche aus ganz Berlin, Brandenburg und dem weiteren Bundesgebiet auf dem Alexanderplatz treffen, wird zum Teil auf Defizite in den »Herkunftssozialräumen « gewertet. Dabei ist die Erweiterung des eigenen Handlungsraums als ein wichtiger Teil adoleszenter Entwicklung anzusehen (Deinet 2009: 23). Dies zeigt sich darin, dass sich Heranwachsende vielfach nicht mehr im Wohnumfeld aufhalten, sondern sich entlang ihrer Interessen attraktive Aufenthalts- und Begegnungsorte suchen, verstärkt durch den Ablösungsprozess vom Elternhaus und unabhängig von der Qualität der Beziehung zu den Eltern.

Junge Menschen eignen sich Räume an, die die Grenzen der sozialräumlichen Logik der Kinder- und Jugendhilfe weit hinter sich lassen. Bei der Planung von Kinder- und Jugendarbeit sorgt dies immer wieder für Irritationen, nicht zuletzt bei der Frage der Zuständigkeit für sozialraumübergreifende Angebote und deren Finanzierung.

\section{Innerstädtische Plätze und Gemeinwesenorientierung}

Nur ein kleiner Anteil der Menschen, die den Alexanderplatz täglich nutzen, wohnen auch dort. Anders als in anderen Stadtteilen werden die Anwohnerinnen und Anwohner als Nutzergruppe kaum wahrgenommen, die Gestaltung des Platzes ist auch kaum auf sie ausgerichtet. Typisch für einen innerstädtischen Platz nutzen die Menschen ihn als Durchgangs- oder vorübergehenden Aufenthaltsort. Konzepte sozialraumorientierter Sozialer Arbeit, die von einer relativ gleichbleibenden und überschaubaren Nachbarschaft als Nutzergruppe ausgehen, laufen hier ins Leere.

Diese besonderen Nutzungsstrukturen eines städtischen Platzes sollten nicht als Störfaktor für eine eng auf die Logik der Sozialraumorientierung ausgerichteten Jugendarbeit angesehen werden. Junge Menschen auf dem Alexanderplatz bilden eher mehrere kategoriale als ein territoriales Gemeinwesen ab. Eine gelingende gemeinwesenorientierte Jugendarbeit muss die Grenzen des verdinglichten Sozialraums überdenken, die Attraktivität dieses Platzes nutzen und fördern und ihre Konzepte und Methoden einer Zielgruppe mit hoher Fluktuation anpassen.

\section{Teilhabe an räumlicher, kultureller und politischer Öffentlichkeit}

Jugendarbeit im öffentlichen Raum darf sich nicht nur dort engagieren, wo sie in der Regel angefragt wird: in sogenannten sozialen Brennpunkten oder dort, wo sie eine Störung durch junge Menschen beenden soll. Vielmehr ist Jugendarbeit gefordert, in attraktiven innerstädtischen Räumen aktiv zu werden, die sich junge Menschen jenseits pädagogischer Aufsicht aneignen und in denen sie in der Regel nur wenig erwünscht sind und Gefahr laufen, verdrängt zu werden.

Das Mandat der Sozialen Arbeit in diesem Feld sollte nicht primär die Befriedung einer konflikthaften Situation umfassen, sondern in einer positiven Wendung junge Menschen im Sinne des \ 11 SGB VIII in ihren Interessen und ihrer (räumlichen) Entwicklung unterstützen.

Gemeinwesenorientiert zu arbeiten bedeutet hier auch, auf die Teilhabe junger Menschen an Öffentlichkeit abzuzielen: im räumlichen Sinn durch die eigenständige Aneignung urbaner Räume und im kulturell-politischen Sinn durch die Mitwirkung an öffentlichen Diskursen und Entscheidungen.

\section{Literatur}

Bingel, Gabriele (2008): Gesellschaftliche Lebensräume für Jugendliche. Aufwachsen in einer Dynamik zwischen Raumzuweisung und Raumaneignung. In: dies./Nordmann, Anja/ Münchmeier, Richard (Hg.): Die Gesellschaft und ihre Jugend. Strukturbedingungen jugendlicher Lebenslagen. Opladen und Farmington Hills: Verlag Barbara Budrich. S. 95-112.

Deinet, Ulrich (2009): Sozialräumliche Aneignung und die Bedeutung des öffentlichen Raums für Jugendliche. In: ders./Okroy, H./ Dodt, G./Wüsthof, A. (Hg.): Betreten Erlaubt! Projekte gegen die Verdrängung Jugendlicher aus dem öffentlichen Raum. Opladen und Farmington Hills: Verlag Barbara Budrich. S. 13-28. Homann, Katharina/Steckelberg, Claudia (2010): Aktuelle Situation im Sozialraum Alexanderplatz aus Sicht junger Menschen eine lebensweltorientierte Erhebung. Internet http://moabiter-ratschlag.de/fileadmin/ dateien/Kinder-_und_Jugendbeteiligung/Platzmanagemment/Bericht_Erhebung_Alexanderplatz.pdf. Abruf vom 4.11.2011.

Nissen, Ursula (1998): Kindheit, Geschlecht und Raum. Sozialisationstheoretische Zusammenhänge geschlechtsspezifischer Raumaneignung. Weinheim und München: Juventa Verlag. Reutlinger, Christian (2005): Gespaltene Stadt und die Gefahr der Verdinglichung des Sozialraums. In: Projekt »Netzwerke im Stadtteil « (Hg.): Grenzen des Sozialraums. Kritik eines Konzepts - Perspektiven für Soziale Arbeit. Wiesbaden: VS Verlag. S. 87-108. 